sepsis, type of feeding and neonatal complications). Information concerning the development of atopy in children in both groups, as well as any family history of atopy, was obtained through a simple questionnaire.

Results There were 38 infants in the group treated with ranitidine (exposure group) and 37 in the control group. There was no significant difference in perinatal characteristics and neonatal morbidity between the two groups. The prevalence of atopy was 39\% in the exposure group and $47 \%$ in the control group $(p=0.57)$. Subgroup analysis showed the prevalence of milk allergy was $17 \%$ in both groups and Non Significant (NS); the prevalence of atopic eczema was $18 \%$ (exposure) vs $45 \%$ (control) $(p=<0.001$ ); and the prevalence of recurrent wheeze or asthma was $13 \%$ (exposure) vs $11 \%$ (control) (NS). Prevalence of other food allergies was very low and comparable. No difference in the family history of atopy was observed.

Conclusions Ranitidine treatment in preterm infants did not increase the overall prevalence of atopy at two years of age but a significantly lower prevalence of atopic dermatitis was observed in the group treated with ranitidine.

\section{G91 A RETROSPECTIVE STUDY OF CHILDREN WITH ACUTE ENCEPHALITIS}

doi:10.1136/archdischild-2013-304107.103

K Eayrs, H Edmond, D Kelly. Department of Paediatrics, Oxford University Hospitals, Oxford, UK

Background and Aims Encephalitis is a relatively rare but potentially devastating condition ${ }^{1}$. Our study investigated the presentation and management of children with encephalitis presenting to a tertiary UK hospital.

Methods Cases of encephalitis presenting during 2001 - 2009 were identified using clinical coding records and a clinical database maintained by the paediatric infectious diseases team. Inclusion criteria were children between the ages of 3 months to 16 years who presented with an acute encephalopathy. A retrospective case notes review was performed and management compared to local infectious disease guidelines for children with an unexplained acute encephalopathy.

Results 71 cases were identified and notes were found in 58 cases. 29 cases met the study criteria.

The commonest presenting features were confusion (86\%), fever $(69 \%)$, seizures (62\%) and headache (60\% in those aged over 5 years).

Microbiological and metabolic workup was inconsistent in relation to the local hospital guidelines. In particular exclusion of metabolic causes of acute encephalopathy was fully undertaken in only $28 \%$ of children. Confirmed or probable infectious aetiologies were identified in 21 cases (72\%) with HSV (17\%), VZV (10\%) and EBV $(7 \%)$ being the most commonly identified pathogens. Other final diagnoses included Acute Disseminated Encephalomyelitis $(14 \%)$.

With regards to treatment, $97 \%$ of children were started empirically on aciclovir although $43 \%$ were prescribed half or less of the recommended dose for encephalitis. Anti-microbial use was also inconsistent between cases: ceftriaxone was started in $76 \%$, azithromycin in $34 \%$ and amoxicillin in $12 \%$.

Conclusion The initial investigation and management of children with an acute encephalopathy who are subsequently diagnosed with encephalitis did not fully follow the guideline in $76 \%$ of cases. The most important finding was the high incidence of inadequate aciclovir dosing in the empirical treatment of children with suspected encephalitis. Recently published guidance highlights the importance of a full clinical, microbiological and metabolic work up and emphasises the correct aciclovir dose ${ }^{1}$. Although encephalitis is an uncommon condition, awareness of its management is vital to prevent the high morbidity associated with the disease.

\section{REFERENCE}

1. Thompson C, Kneen R, Riordan A, Kelly D, Pollard AJ. Encephalitis in children. Arch Dis Child. 2012 Feb; 97(2):150-61.

\section{G92 PRESCRIPTIONS OF ORAL PENICILLINS FOR CHILDREN: EVIDENCE OF WIDESPREAD DOSING VARIATION IN PRIMARY CARE}

doi:10.1136/archdischild-2013-304107.104

1,2S Saxena, ${ }^{3} \mathrm{Z}$ Ismael, ${ }^{4} \mathrm{CIS}$ Barker, ${ }^{5} \mathrm{ML}$ Murray, ${ }^{6} \mathrm{CK}$ Wong, ${ }^{7} \mathrm{M}$ Sharland, ${ }^{3,8 \mathrm{PF}}$ Long ${ }^{1}$ Chartfield Surgery, Putney, London, UK; ${ }^{2}$ Department of Primary Care and Public Health, Imperial College London, London, UK; 'Institute of Pharmaceutical Science, King's College London, London, UK; 'Paediatric Infectious Diseases Research Group, St George's, University of London, London, UK ${ }^{5}$ Centre for Paediatric Pharmacy Research, University College London School of Pharmacy, London, UK; ${ }^{6}$ Department of Pharmacology and Pharmacy, The University of Hong Kong, Pokfulam, Hong Kong, China'Paediatric Infectious Diseases Unit, St George's Hospital, London, UK: ${ }^{8}$ Department of Chemistry, King's College London, London, UK

Aims The British National Formulary for Children (BNFC) provides recommendations for dosing oral penicillins in children according to age-bands, weight-bands, and weight-based calculations. However, as childhood obesity is increasingly prevalent, dosing by age-bands could lead to clinically significant under-dosing. This study aimed to determine how current UK GP prescribing practise follows the original age-band dosing recommendations, which could lead to sub-therapeutic dosing.

Methods Detailed prescriptions for the oral penicillins in 0-18 year-old children from 2010 were analysed from the IMS DiseaseAnalyzer database, which contains computerised medical records from 125 general practises, representing approximately $2 \%$ of the UK population. It includes data on formulation, strength, prescription quantity unit, package size, prescribed quantity and volume.

Results For 2010, 388,926 patients aged 0-18 years were registered on the database (51\% male), accounting for 376,292 person-years in total. There were 65,737 prescriptions for oral penicillins identified: amoxicillin (63\%), penicillin $\mathrm{V}(17 \%)$ and flucloxacillin $(20 \%)$.

The amoxicillin prescription results by age-band (as an example) showed:

1. In the first age-band of less than 1 year, no child received an amoxicillin prescription for the recommended unit dose of $62.5 \mathrm{mg}$; the majority received double the unit dose $(125 \mathrm{mg})$;

2. In the second age-band of 1 to 5 years, $96 \%$ received prescriptions for the recommended unit dose $(125 \mathrm{mg})$;

3. $40 \%$ of $6-12$ year-olds and $70 \%$ of $12-18$ year-olds were prescribed unit doses below those recommended in the BNFC for their age-band.

Prescriptions for otitis media were analysed separately. As patient weight was not available, average weights were used for the analysis (based on the 2010 Health Survey of England), and the dose in $\mathrm{mg} / \mathrm{kg} /$ day was calculated. From these data, only children less than 1 year received the recommended BNFC dose of $40-90 \mathrm{mg} / \mathrm{kg} /$ day. For children aged $4-15$ years, the prescriptions equated to $10-20 \mathrm{mg} / \mathrm{kg} /$ day, approximately $33 \%$ of the recommended dose.

Conclusions These results demonstrate wide variation in the dosing of penicillins for children in primary care. There is an urgent need to review and simplify current dosing ecommendations according to age-bands and weight-bands, in relation to the average weights of children today. 